\title{
EDITORIAL Sagittal craniosynostosis: what matters to parents?
}

\author{
Ann-Christine Duhaime, MD \\ Pediatric Neurosurgery, Massachusetts General Hospital, Boston, Massachusetts
}

$\mathrm{I}$ $\mathrm{N}$ their article "Sagittal craniosynostosis: a utility outcomes study," Kuta and colleagues used health state utility scores to compare the perceived burden of sagittal synostosis as a health care condition relative to monocular or binocular blindness. " Their goal was "to better understand the conflict felt by families when offered a potentially morbid corrective surgery in a child's 1st year of life for an aesthetic indication." This is a worthy goal, and surgeons should participate in and make use of the collection of data that can better inform them about what actually matters to patients. As in all research, understanding the methods may be helpful to put the results in context.

The survey utilized in this study asked adults without the condition, who had been recruited from the general public, as well as medical students (36.4\% of study participants) to imagine what it would be like to have the condition and to rate the health-related quality of life. In the case of blindness, one can assume that most people can imagine what living without eyesight in one or both eyes might involve. In the case of sagittal synostosis, most of what participants might know about the condition comes primarily from what the researchers provide as information. In this study, to imagine the impact of scaphocephaly, subjects were provided anteroposterior and lateral photographs of a baby with this condition, and the authors note that participants were provided information that the condition might have the potential to progress. The authors found that scaphocephaly was perceived as having less effect on quality of life than monocular blindness; thus, they concluded that the burden of disease is low and "should inform surgeons" discussions when offering morbid corrective surgery, particularly when driven by aesthetic concerns."

The authors acknowledge that their approach does not ask patients with treated or untreated sagittal synostosis or their families, what is their impression of quality of life. Nor does their method assess participants' understanding of the actual potential effect or range of disability associated with this condition, especially since their understanding is primarily provided by two photographs of an appealing baby with relatively mild scaphocephaly prior to progression. Many neurosurgeons have encountered sagittal syn- ostosis infants whose own parents did not fully appreciate the extent of the condition until it was demonstrated with photographs or other teaching aids; babies are appealing by design, even with scaphocephaly. The study did not include a photograph of an adolescent or adult with untreated sagittal synostosis. It did not ask subjects to imagine difficulties finding sports helmets that fit or scenarios of being teased by other children. It did not mention the comparative burden and risk of the major cranial vault reconstruction necessary for correction if a decision to proceed is delayed to later childhood. Further, the authors did not mention the possibility of visual loss from untreated sagittal synostosis with increased intracranial pressure, which is known to occur in some children with this condition when untreated and which can be difficult to predict. Thus, this behavioral economic approach to a condition about which subjects have limited knowledge may add some information about what people may believe relative to other conditions that they can more easily imagine, but it does not directly measure the burden to patients with the condition and their families, especially when additional potential consequences like visual loss are not included.

Approaches using this general, economic-type decision tool to measure an index of relative value or worth, when the opinions garnered depend so heavily on the specific information provided, offer some useful perspective, but also have some hazards. One cannot use data from this type of study to assume that conditions such as sagittal synostosis are unlikely to be meaningful or matter to parents and children. Surgeons are held to a high standard of fully describing goals, risks, and alternatives to any surgical intervention. Descriptions of surgery should cover common and less common risks, short- and long-term outcomes, and the pros and cons of alternative surgical approaches. Discussions of conservative management should cover potential evolution and complications over time, with estimates based on the best data on the likelihood of each outcome or risk of progression. For each family, these various risks and consequences may resonate differently. Therefore, while studies such as this one may be useful, they provide an incomplete shortcut to understanding dis- 
ease burden, when measuring what people with limited information might imagine the case to be. It is challenging to thoroughly counsel families whose children have conditions with which they are probably unfamiliar and that may be treated with potentially morbid surgery. Studies such as this one may increase surgeons' awareness of the perceptions of the general public, as a useful starting point. Thorough discussions - often several, repeated over time-reviewing goals, risks, and alternatives, covering short- and long-term aesthetic and functional outcomes as well as surgical and conservative management, and addressing specific questions raised, remain the primary tool to help families assess their own sense of how best to optimize health care quality of life for their children.

https://thejns.org/doi/abs/10.3171/2017.3.PEDS1758

\section{References}

1. Kuta V, McNeely PD, Walling S, Bezuhly M: Sagittal craniosynostosis: a utility outcomes study. J Neurosurg Pediatr [epub ahead of print May 19, 2017. DOI: 10.3171/2017.2.PEDS16567]

\section{Disclosures}

The author reports no conflict of interest.

\section{Response}

\section{Victoria Kuta, BScH, ${ }^{1}$ P. Daniel McNeely, MD, FRCSC, ${ }^{2}$ Simon Walling, MBChB, FRCSC, ${ }^{2}$ and Michael Bezuhly, MD, FRCSC ${ }^{3}$}

${ }^{1}$ Faculty of Medicine; ${ }^{2}$ Division of Neurosurgery, IWK Health Centre; and 'Division of Plastic \& Reconstructive Surgery, IWK Health Centre, Dalhousie University, Halifax, Nova Scotia, Canada

We thank Dr. Duhaime for her insightful comments regarding our article. As she rightly points out, our study provides useful information to surgeons by underscoring the potential for decisional conflict among parents of infants with sagittal craniosynostosis. Our findings are not altogether surprising. When presented with photographs of an appealing, developmentally normal baby with relatively mild scaphocephaly, members of the general public rated the burden of sagittal craniosynostosis as low. Although in the presented scenario we informed respondents that the head shape would worsen with time, we did not provide photographs of an untreated adolescent. Instead, we presented the situation in which parents would find themselves prior to seeing a surgeon for the first time. The intent of our study was to raise awareness of parental decisional conflict so that surgeons can be better equipped to address the concerns of families during the initial consultation and informed consent process.

Dr. Duhaime's caveat regarding the over-interpretation of our findings is a critical one. Our study does not claim that sagittal craniosynostosis is a low-burden health state, particularly to those families affected by it. Children with progressive scaphocephaly can be the target of bullying because of their appearance or can have functional concerns such as an inability to find proper-fitting helmets for sports activities. In rare cases, sagittal craniosynostosis can lead to increased intracranial pressure and visual loss.

Despite its limitations, we feel that our study represents an important first step in exploring the health burden associated with sagittal craniosynostosis. As Dr. Duhaime has intimated, a simple next step would be to assess the health state utility scores reported by patients and families with sagittal craniosynostosis. Additionally, our group is using qualitative methods to interview parents of children newly diagnosed with sagittal craniosynostosis to identify common themes and concerns. Ultimately, by gaining an appreciation of how families experience a given condition, surgeons will be better positioned to provide patient-centered care. 